Pacific

Journal of

Mathematics

ON THE ACTION OF THE GROUP OF DIFFEOMORPHISMS OF A SURFACE ON SECTIONS OF THE DETERMINANT LINE BUNDLE

Doug PiCKRell 


\title{
ON THE ACTION OF THE GROUP OF DIFFEOMORPHISMS OF A SURFACE ON SECTIONS OF THE DETERMINANT LINE BUNDLE
}

\author{
Doug PiCKRELL
}

\begin{abstract}
Let $\Sigma$ denote a closed oriented surface. There is a natural action of the group $\operatorname{Diff}^{+}(\Sigma)$ on sections of the chiral determinant line over the space of gauge equivalence classes of connections. The question we address is whether this action is unitarizable. We introduce a $S \mathrm{D} i f f$-equivariant regularization, and we prove the existence of, and explicitly compute, the limit as the regularization is removed. The $S D i f f$ unitary representations that arise, both by regularization and after removing the regularization, appear to be new.
\end{abstract}

\section{Introduction.}

Let $\Sigma$ denote a closed oriented surface, and let $\mathcal{D}$ denote the group of orientation-preserving diffeomorphisms of $\Sigma$. Let $K$ denote a connected compact Lie group, $\mathcal{A}$ the space of $K$-connections in the trivial bundle $P=(\Sigma \times K \rightarrow \Sigma)$, and $\mathcal{C}$ the space of gauge equivalence classes of $K$ connections. It is well-known that the orientation of $\Sigma$ induces a $\operatorname{Aut}(P)$ invariant symplectic structure on $\mathcal{A}$. Ideally we would want to consider the action of $\mathcal{D}$ in the metaplectic representation, corresponding to a polarization coming from a choice of complex structure for $\Sigma$, but it is easy to check that $\mathcal{D}$ is not implemented in this representation (see $\S 1$ ); instead $\mathcal{D}$ permutes these representations. Roughly speaking, our goal is to understand whether the gauge invariant sectors of these representations (the conformal blocks of conformal field theory) can be coherently incorporated into a single unitary representation for $\mathcal{D}$.

To be more precise, there exists a nontrivial $\mathcal{D}$-equivariant Hermitian line bundle $\mathcal{L} \rightarrow \mathcal{C}$ (the projection to $\mathcal{C}$ of the prequantum line bundle on $\mathcal{A}$ ). The issue we address is whether, in the simplest case $K=\mathbb{T}$, a submodule of the natural action

$$
\mathcal{D} \times \Omega^{0}(\mathcal{L}) \rightarrow \Omega^{0}(\mathcal{L})
$$

can be unitarized. Heuristically the invariant inner product is given by

$$
s_{1}, s_{2} \rightarrow \int_{\mathcal{C}}\left\langle s_{1}, s_{2}\right\rangle d V,
$$


where " $d V$ " is a fictitious $\mathcal{D}$-invariant measure on $\mathcal{C}$ arising from the $\mathcal{D}$ invariant symplectic structure on $\mathcal{A}$.

The submodule arises in the following way. Fix a complex structure on $\Sigma$. We can then identify $\mathcal{L}$ with a determinant line bundle for $\bar{\partial}$ coupled to gauge potentials. There is a canonical section det which is fixed by the subgroup $\operatorname{Aut}(\Sigma) \subset \mathcal{D}$. The submodule is the spherical representation for the pair $(\mathcal{D}, \operatorname{Aut}(\Sigma))$ generated by det.

To obtain a unitary structure we choose a positive area form on $\Sigma$, and introduce a regularization, in this case the Yang-Mills measure on (a completion of) $\mathcal{C}$

$$
d \nu_{T}([A])=\frac{1}{\mathcal{Z}(T)} e^{-\frac{1}{2 T} \int\left\langle F_{A} \wedge * F_{A}\right\rangle} d V([A]) .
$$

For each $T<\infty$, we rigorously obtain a $S$ Diff-invariant unitary structure

$$
s_{1}, s_{2} \rightarrow\left\langle s_{1}, s_{2}\right\rangle_{T}=\int s_{1} \bar{s}_{2} d \nu_{T}^{|\mathcal{L}|^{-2}}
$$

where $d \nu_{T}^{|\mathcal{L}|^{-2}}$ denotes the renormalized coupling of the Yang-Mills measure and Hermitian structure of $\mathcal{L}$. In the genus $=0$ case, the spherical function

$$
\phi_{T}: \mathcal{D} / \operatorname{Aut}(\Sigma) \rightarrow \mathbb{C}: \sigma \rightarrow\langle\sigma \cdot \operatorname{det}, \operatorname{det}\rangle_{T}
$$

is given by the formula

$$
\phi_{T}(\sigma)=\operatorname{det}_{2}\left(1+T(\partial \bar{\partial}-\partial \mu \partial)^{-1} *\right)^{-1 / 2},
$$

where $\mu$ is the complex dilatation of $\sigma^{-1}$ (see $\S 1$ ), and $\operatorname{det}_{2}$ denotes the Hilbert-Schmidt regularized determinant. Although $\phi_{T}$ is defined on all of $\mathcal{D}$, only its restriction to $S$ Diff is positive definite.

The main result of this paper is that one can remove the regularization. After renormalizing $\phi_{T}$, one can take the limit $T \rightarrow \infty$ to obtain (in the genus $=0$ case) a positive definite function

$$
\phi: S \text { Diff } \rightarrow \mathbb{C}: \sigma \rightarrow \frac{\operatorname{det}_{\zeta}\left(4 \Delta\left(1-\bar{\partial}^{-1} \mu \partial\right)^{-1}\right)^{-1 / 2}}{\operatorname{det}_{\zeta}(4 \Delta)^{-1 / 2}}
$$

(the precise meaning of this formula is explained in $\S 3$ ). One can heuristically arrive at this formula for $\phi$ by using $\zeta$-function determinants to directly "evaluate" certain integrals. On the other hand it is not at all transparent that $|\phi|$ is bounded by 1 on SDiff, let alone that $\phi$ is a positive definite function on $S$ Diff; this follows by first evaluating the regularized integrals and then taking the limit $T \rightarrow \infty$. From a purely technical point of view, one of the most interesting aspects of this work is the following: There are two regularizations involved in arriving at (0.6), one probabilistic and one of the $\zeta$ variety, and it is fascinating to see how they balance out (see Remarks $(2.28)$ and $(3.30))$. 
To go from (0.5) to (0.6), we establish a result of independent interest concerning the multiplicative anomaly for $\zeta$-function determinants. If $E$ is a classical, elliptic, invertible pseudo-differential operator on an $n$ dimensional compact manifold, with symbol satisfying the Agmon-Niremberg condition, and if $C$ has order $<-n$, so that it represents a trace class operator, then it is known that

$$
\operatorname{det}_{\zeta}\left(E e^{\tau C}\right)=\operatorname{det}_{\zeta}(E) e^{\tau \operatorname{tr}(C)},
$$

as one would naively suspect $([\mathbf{K V}])$. We consider the borderline case in which $C$ has order $-n$, in which case the trace is replaced by a regularized trace, depending upon $E$; see Lemma (3.10) for the precise statement.

The formula (0.6) defines an extension of $\phi$ to all of $\mathcal{D}$, but this extension cannot possibly be positive definite; for (0.6) defines a holomorphic function of $\mu$, hence cannot possibly be bounded by 1 . This strongly suggests that the action (0.1) is not unitarizable, so that the answer to our original question is negative. We should mention that this question cannot be decided by considering the Lie algebra action, for det cannot possibly be a differentiable vector in a unitary representation for $\mathcal{D}$ (see $\S 1$ ).

The theory of unitary representations for various types of diffeomorphism groups is basically at the stage of searching for interesting examples; we refer to $[\mathbf{I}]$ for an account of this. It appears that the unitary representations of SDiff which we have constructed, both the regularized representations and their limit, are new (but, as pointed out by the referee, it is difficult to judge this from the spherical function alone). Because we assume $K=\mathbb{T}$ is abelian, the Yang-Mills measure (0.2) is essentially Gaussian, so that it is a fairly mundane object. However, we should point out that in 2 dimensions, the Yang-Mills measure is well-defined mathematically for any compact Lie group $K$ (see $[\mathbf{S e n}]$ or $[\mathbf{P i}]$ and references there), so that the corresponding representations should exist in this more general (and technically challenging) setting. In terms of mathematical physics, the regularized representations amount to a geometric formulation of the coupling of Yang-Mills fields to massless fermions (see $[\mathbf{P i}]$ ). The unitary structure that should arise when the regularization is removed should restrict to a canonical scalar product on the spaces of conformal blocks in conformal field theory (or equivalently the space of states for Chern-Simons theory), the existence of which has been proven in some nonabelian cases by Gawedzki (see $[\mathbf{G}]$ and references there). As we mentioned previously, understanding whether these blocks fit together coherently to give a unitary representation of $\mathcal{D}$ was the original motivation for this work.

The outline of the paper is as follows. In $\S 1$ we briefly review what we need to know from the theory of quasiconformal mappings, and the relation of this to the symplectic action of $\mathcal{D}$ on the space of all connections. In $\S 2$ and $\S 3$ we deal with the regularized and limiting representations in the 
genus $=0$ case. In the last section we indicate the relatively minor changes necessary to deal with the cases of positive genus.

Acknowledgement. I thank Lennie Friedlander and John Palmer for useful discussions on determinants. I also thank the referee for useful comments.

\section{The Quotient $\mathcal{D} / \operatorname{Aut}(\Sigma)$.}

Given a closed oriented surface $\Sigma$, there is a natural symplectic structure $\omega$ on the real vector space $\Omega^{1}(\Sigma)$ of one-forms on $\Sigma$ :

$$
\omega(\theta, \eta)=\int_{\Sigma} \theta \wedge \eta
$$

The choice of a complex structure $j$ on $\Sigma$ induces a positive polarization:

$$
\left(\Omega^{1}\right)^{\mathbb{C}}=\Omega^{0,1} \oplus \Omega^{1,0} .
$$

Let $\sigma$ denote an orientation-preserving diffeomorphism of $\Sigma$. Following standard practice, with respect to the decomposition (1.2), we write

$$
\mathcal{D} \rightarrow S p\left(\Omega^{1}, \omega\right): \sigma \rightarrow\left(\sigma^{-1}\right)^{*}=\left(\begin{array}{cc}
A & B \\
\bar{B} & \bar{A}
\end{array}\right),
$$

where we have extended $\left(\sigma^{-1}\right)^{*}$ by complex linearity to $\left(\Omega^{1}\right)^{\mathbb{C}}$.

Given a general symplectic space $(V, \omega)$, it does not make sense to speak of a bounded symplectic transformation. However, given a positive polarization,

$$
V^{\mathbb{C}}=V^{+} \oplus V^{-}
$$

where $V^{+}$is a complex Hilbert space with respect to the form $i \omega(\theta, \bar{\eta})$, and $V^{-}$is the conjugate Hilbert space, we can define

$$
\begin{aligned}
& S p_{(\mathcal{L})}\left(V^{+} \oplus V^{-}, \omega\right) \\
&(1.5)=\left\{g=\left(\begin{array}{ll}
A & B \\
\bar{B} & \bar{A}
\end{array}\right) \in S p(V, \omega): B \in \mathcal{L}\right\} \\
&(1.6)=\left\{\left(\begin{array}{ll}
A & B \\
\bar{B} & \bar{A}
\end{array}\right) \in \mathcal{L}\left(V^{+} \oplus V^{-}\right): A^{*} A-\bar{B}^{*} \bar{B}=1, A^{*} B-\bar{B}^{*} \bar{A}=0\right\},
\end{aligned}
$$

where " $\mathcal{L}$ " stands for bounded operators, $\bar{B}$ is the conjugate of $B$, and the adjoint is computed with respect to the Hilbert space structure of $V^{+} \oplus$ $V^{-}$. We then have a fibration over a (non-Riemannian) symmetric bounded domain

$$
0 \rightarrow U\left(V^{+}\right) \rightarrow S p_{(\mathcal{L})} \rightarrow\left\{Z \in \mathcal{L}\left(V^{+}, V^{-}\right): Z=\bar{Z}^{*}, Z^{*} Z<1\right\} \rightarrow 0
$$


where $A \rightarrow\left(\begin{array}{cc}A & \\ & \bar{A}\end{array}\right), g \rightarrow Z$,

$$
g=\left(\begin{array}{ll}
A & B \\
\bar{B} & \bar{A}
\end{array}\right)=\left(\begin{array}{ll}
1 & \\
Z & 1
\end{array}\right)\left(\begin{array}{ll}
A & \\
& \bar{A}-Z A W
\end{array}\right)\left(\begin{array}{cc}
1 & W \\
& 1
\end{array}\right) .
$$

It is more common to consider proper operator ideals, especially HilbertSchmidt operators in representation-theoretic contexts, in place of $\mathcal{L}$ above, but our point will be that $\mathcal{L}$ is natural in our context.

To apply this to our context, let $V$ denote the completion of $\Omega^{1}$ with respect to our chosen complex structure on $\Sigma$,

$$
V=\left\{\theta \in \Omega_{\text {meas }}^{1}: \int \theta \wedge * \theta<\infty\right\} .
$$

The form $\omega$ extends continuously to $V$, and we let $V^{+}$denote the completion of $\Omega^{0,1}$.

Proposition 1.10. The map (1.3) extends to a representation of the group of quasi-conformal homeomorphisms

$$
\mathcal{D}_{q c} \rightarrow S p_{(\mathcal{L})}\left(V^{+} \oplus V^{-}, \omega\right) .
$$

In the genus $=0$ case, the fibration (1.7) induces an exact sequence

$$
0 \rightarrow \operatorname{Aut}(\Sigma) \rightarrow \mathcal{D}_{q c} \rightarrow \Omega_{L_{<1}^{\infty}}^{0}\left(T^{* 0,1} \otimes T^{1,0}\right) \rightarrow 0,
$$

where $\sigma \rightarrow \mu$, the complex dilatation of $\sigma^{-1}$, and $\bar{Z}(\sigma)=\mu=-W(\sigma)$, where $\mu$ is viewed as a multiplication operator (see Remark (2) below); in general we have an exact sequence

$$
0 \rightarrow \operatorname{Aut}(\Sigma) \rightarrow \mathcal{D}_{q c} \rightarrow \Omega_{L_{<1}^{\infty}}^{0}\left(T^{* 0,1} \otimes T^{1,0}\right) \rightarrow \mathcal{M}(\Sigma) \rightarrow 0,
$$

where $\mathcal{M}(\Sigma)$ is the moduli space of Riemann surface structures for $\Sigma$, with basepoint $j$.

Remarks 1.11. (1) Note that there is a $\operatorname{Aut}(\Sigma)$-equivariant mapping

$$
\Omega^{0}\left(T^{* 0,1} \otimes T^{1,0}\right) \rightarrow \Omega^{0}: \mu \rightarrow \mu \otimes \bar{\mu}
$$

so that there is a $\operatorname{Aut}(\Sigma)$-invariant $L^{\infty}$ structure on $\Omega^{0}\left(T^{* 0,1} \otimes T^{1,0}\right)$. In (1.10) " $L_{<1}^{\infty}$ " denotes the unit ball with respect to this Banach structure.

(2) Given $\mu \in \Omega_{L^{\infty}}^{0}\left(T^{* 0,1} \otimes T^{1,0}\right)$, there is an associated bounded "multiplication operator"

$$
\mu: V^{-} \rightarrow V^{+}: \theta \rightarrow \mu \otimes \theta
$$

Using the coordinate expressions, $\theta=f d z, \mu=\tilde{\mu} d \bar{z} \otimes \frac{\partial}{\partial z}$, and $\mu \otimes \theta=\tilde{\mu} f d \bar{z}$, it is obvious that $|\mu|_{\mathcal{L}}=|\mu|_{L^{\infty}}$. 
(3) A homeomorphism $\sigma$ is quasi-conformal provided (1) in holomorphic coordinates $\sigma$ has locally square-integrable partial derivatives, and (2) with respect to the decomposition $T^{\mathbb{C}}=T^{1,0} \oplus T^{0,1}$, if

$$
(d \sigma)^{\mathbb{C}}=\left(\begin{array}{cc}
\partial \sigma & \bar{\partial} \sigma \\
(\bar{\partial} \sigma)^{*} & (\partial \sigma)^{*}
\end{array}\right),
$$

then the complex dilatation

$$
\mu_{\sigma}=\frac{\bar{\partial} \sigma}{\partial \sigma} \in \Omega^{0}\left(T^{* 0,1} \otimes \sigma^{*} T^{1,0} \otimes\left(T^{* 1,0} \otimes \sigma^{*} T^{1,0}\right)^{-1}\right)=\Omega^{0,1}\left(T^{1,0}\right)
$$

satisfies $\left|\mu_{\sigma}\right|_{L^{\infty}}<1$.

Proof of (1.10). Suppose $\sigma$ is quasi-conformal. Then $\sigma^{-1}$ is quasi-conformal, and it is straightforward to show that

$$
W(\sigma)=-\mu, \quad Z(\sigma)=\bar{\mu} .
$$

For example to see that $W(\sigma)=-\mu$, suppose that $\theta \in \Omega^{1,0}$. Since $W=$ $A^{-1} B$, we must show that

$$
B \theta=-A \mu \theta, \quad \text { i.e., } \quad\left(\sigma^{*} \theta\right)^{0,1}=-\left(\sigma^{*}(\mu \theta)\right)^{0,1} .
$$

In local coordinates $w=\sigma(z), \theta=f d w, \mu=\mu_{\sigma^{-1}}=\frac{z_{\bar{w}}}{z_{w}} d \bar{w} \otimes \frac{\partial}{\partial w}$, and

$$
\left(\left(\begin{array}{ll}
z_{w} & z_{\bar{w}} \\
\bar{z}_{w} & \bar{z}_{\bar{w}}
\end{array}\right) \circ \sigma\right)\left(\begin{array}{ll}
w_{z} & w_{\bar{z}} \\
\bar{w}_{z} & \bar{w}_{\bar{z}}
\end{array}\right)=\left(\begin{array}{ll}
1 & \\
& 1
\end{array}\right) \cdot
$$

The LHS of (1.16) equals $f(\sigma(z)) w_{\bar{z}} d \bar{z}$, and the RHS of (1.16) equals

$$
-\left(\frac{z_{\bar{w}}}{z_{w}}\right) \circ \sigma(z) \bar{w}_{\bar{z}} f(\sigma(z)) d \bar{z} .
$$

Thus (1.16) follows from the $(1,2)$ entry of $(1.17)$.

Thus $\sigma$ quasi-conformal implies $Z=\bar{\mu}$ is bounded and $|Z|_{\mathcal{L}}<1$. Since $A A^{*}=\left(1-Z Z^{*}\right)^{-1}, A$ and $B$ are also bounded, and it follows that $\sigma \in S p_{(\mathcal{L})}$.

Now suppose that we are given $\mu$ with $|\mu|_{L^{\infty}}<1$. The basic fact in the theory of quasi-conformal mappings is that there exists a (possibly different) complex structure $j^{\prime}$ on $\Sigma$ and a quasi-conformal mapping $\sigma:\left(\Sigma, j^{\prime}\right) \rightarrow$ $(\Sigma, j)$ such that $\mu=\mu_{\sigma^{-1}}$ (see Theorem 1.1 of Chapter $\mathrm{V}$ of $[\mathbf{L}]$ ). In the genus $=0$, case $j^{\prime}$ is equivalent to $j$, and we obtain the first exact sequence in (1.10); in general we obtain the second sequence in (1.10), where $\mu$ maps to the equivalence class of $j^{\prime}$. This completes the proof.

In the introduction we mentioned that the representations we consider do not induce representations of Lie algebras. One way to see this, in the genus $=0$ case, is as follows.

Proposition 1.19. Suppose that $\Sigma=\hat{\mathbb{C}}$, the Riemann sphere. Suppose that

$$
\pi: \mathcal{D} \rightarrow U(H)
$$


is a unitary representation, and suppose also that there exists a smooth vector $v \in H$ which is fixed by $\operatorname{Aut}(\Sigma)=P S L(2, \mathbb{C})$. Then $v$ is fixed by all of $\mathcal{D}$.

Proof. Given that $v$ is fixed by $\operatorname{Aut}(\Sigma)$, there is a mapping

$$
\mathcal{D} / \operatorname{Aut}(\Sigma) \rightarrow \mathbb{P}(H):[g] \rightarrow \mathbb{P}(g \cdot v) .
$$

Since $v$ is a smooth vector, this is a differentiable mapping, and hence we can pull the Fubini-Study metric back to obtain a $\mathcal{D}$-invariant (possibly incomplete) Riemannian structure on $\mathcal{D} / \operatorname{Aut}(\Sigma)$. But by considering the isotropy action of $\operatorname{Aut}(\Sigma)$ at the base point, this means that we have a $\operatorname{Aut}(\Sigma)$-invariant unitary structure for the natural action

$$
\operatorname{Aut}(\Sigma) \times \Omega^{0,1}\left(T^{1,0}\right) .
$$

But it is known that this particular representation for $P S L(2, \mathbb{C})$ is not unitarizable (see e.g. Chapter 1 of $[\mathbf{K}]$ ). Since $\operatorname{sl}(2, \mathbb{C})$ is a maximal closed subalgebra of vect $(\Sigma)$, it follows that $\mathcal{D}$ must act trivially on $v$. This completes the proof.

\section{2. $S \operatorname{Diff}\left(S^{2}\right)$ unitary representations.}

Throughout this section and the next, $\Sigma$ will denote a closed oriented surface of genus $=0$.

Let $\mathcal{A}$ denote the set of continuous $\mathbb{T}$-connections in the trivial bundle $\Sigma \times \mathbb{T} \rightarrow \Sigma$, and let $\mathcal{K}$ denote the $C^{1}$ gauge group. Since genus $(\Sigma)=0, \mathcal{K}$ is connected, hence $\mathcal{K}=\exp \left(\Omega_{C^{1}}^{0}(\Sigma, i \mathbb{R})\right)$. Define

$$
\begin{aligned}
\Omega^{0}(\mathcal{L})=\{f: \mathcal{A} \rightarrow \mathbb{C}: f(A+d \xi)=\exp & \left(i \int A \wedge d \xi\right) f(A) \\
& \left.\forall A \in \mathcal{A}, \xi \in \Omega_{C^{1}}^{0}(\Sigma, i \mathbb{R})\right\} .
\end{aligned}
$$

This is the space of sections of a line bundle $\mathcal{L} \rightarrow \mathcal{A} / \mathcal{K}$, hence the notation. It is easy to check that the natural action by pullback leads to a representation

$$
\mathcal{D} \times \Omega^{0}(\mathcal{L}) \rightarrow \Omega^{0}(\mathcal{L}) .
$$

To unitarize this representation, it would suffice to have a $\mathcal{D}$-invariant measure on the base space $\mathcal{A} / \mathcal{K}$, since $\mathcal{L}$ has a natural Hermitian structure. But such a measure does not exist. The point of this subsection is that this naive idea can be made to work, provided that we choose an area form on $\Sigma$, and restrict the representation to $S$ Diff, the area-preserving diffeomorphisms.

So fix a smooth area form on $\Sigma$. We can then consider the Yang-Mills measure, corresponding to temperature $T$, which is given heuristically by 
the expression

$$
d \nu_{T^{-1} Y M}(A)=\frac{1}{\mathcal{Z}} \exp \left(\frac{1}{2 T} \int F \wedge * F\right) \mathcal{D} A
$$

where $F=d A$ is curvature. In this abelian context it is well-known that one can interpret the projection of the heuristic expression (2.3) to $\mathcal{A} / \mathcal{K}$ as a Gaussian measure, and this Gaussian is SDiff-invariant. By definition the "projection" is the Gaussian measure $\nu_{T}$ corresponding to the real Hilbert space structure

$$
F \cdot_{T} F=\frac{1}{T} F \cdot F=-\frac{1}{T} \int F \wedge * F,
$$

where $F$ is an $i \mathbb{R}$-valued two-form with $\int F=0$. Since $S$ Diff acts orthogonally on this Hilbert space, $\nu_{T}$ is an $S$ Diff-invariant measure. The question that we must address is whether we can use this measure to integrate functions of the form $s_{1} \bar{s}_{2}$, for $s_{1}, s_{2} \in \Omega^{0}(\mathcal{L})$.

The most interesting sections are obtained in the following way. Choose a complex structure for $\Sigma$. We then have an isomorphism

$$
\mathcal{A} \rightarrow \Omega_{C^{0}}^{0,1}: A \rightarrow a=A^{0,1}, \quad A=a-a^{*} .
$$

It is easy to check that the function

$$
\operatorname{det}: \mathcal{A} \rightarrow \mathbb{C}: A \rightarrow \exp \left(i \int\left(a \wedge\left(a^{*}+H a\right)\right)\right)
$$

is in $\Omega^{0}(\mathcal{L})$, where (because genus $\left.(\Sigma)=0\right) H$ is the well-defined unitary $0^{\text {th }}$-order operator

$$
H=\partial \bar{\partial}^{-1}: \Omega_{L^{2}}^{0,1} \rightarrow \Omega_{L^{2}}^{1,0} .
$$

$H$ is often referred to as either the Hilbert or Beurling transform. The rationale for the notation is that, relative to our choice of complex structure, $\mathcal{L}$ can be identified with the determinant line bundle with Quillen metric for the family of Fredholm operators $\bar{\partial}_{\kappa^{1 / 2}}+a, a \in \Omega^{0,1}$, and the canonical section corresponds to the function (2.6).

In order to have a better grasp of the section det, it is useful to consider the parameterization of $\mathcal{A}$

$$
a=\bar{\partial} x, \quad x \in \Omega_{C^{1}}^{0}(\Sigma, \mathbb{C}) / \mathbb{C} .
$$

A gauge transformation $A \rightarrow A+d \xi$ corresponds to $a \rightarrow a+\bar{\partial} \xi$, i.e., adding a $i \mathbb{R}$-valued function to $x$. Hence we can parameterize the space $\mathcal{A} / \mathcal{K}$ by $x \in \Omega_{C^{1}}^{0}(\Sigma, \mathbb{R})$. The relation between ( $\mathbb{R}$-valued) $x$ and the curvature $F$ is simply

$$
F=i * \Delta x=2 \partial \bar{\partial} x
$$


In terms of $x$ the projection of the Yang-Mills measure with temperature $T$ to $\mathcal{A} / \mathcal{K}$ is the Gaussian measure corresponding to the real Hilbert space structure

$$
x \cdot T x=-\frac{1}{T} \int F \wedge * F=\frac{1}{T} \int * \Delta x \wedge \Delta x .
$$

From this we can spot the basic source of difficulty: This Gaussian measure is not supported on $x \in C^{1}$; rather, it is only supported on $x \in C^{0} \cap$ $W^{1-\epsilon}$, for $\epsilon>0$. Hence we cannot integrate functions of the form $s_{1} \bar{s}_{2}$ in a straightforward manner. Essentially what we must do is show that the bundle $\mathcal{L}$ can be $S$ Diff-equivariantly extended to the support of $\nu_{T}$.

In terms of ( $\mathbb{C}$-valued) $x$, the section det is given by the expression

$$
\left.\operatorname{det}\right|_{x}=\exp \left(i \int \bar{\partial} x \wedge 2 \partial(\mathbb{R} e(x))\right)=\exp \left(i \int x \wedge F\right)
$$

where the first equality makes sense if $x \in C^{1}$, the second if $x \in C^{2}$. Given $\sigma \in \mathcal{D}$ we obtain a transition function

$$
c_{\mathcal{A} / \mathcal{K}}(\sigma, x)=\left.\frac{\sigma \cdot \operatorname{det}}{\operatorname{det}}\right|_{x}
$$

for the line bundle $\mathcal{L}$ over $\mathcal{A} / \mathcal{K}$, where we are viewing ( $\mathbb{R}$-valued) $x \in C^{1} / \mathbb{R}$ as a global coordinate. We can also interpret $c_{\mathcal{A} / \mathcal{K}}$ as a cocycle, i.e., it satisfies the equation

$$
c_{\mathcal{A} / \mathcal{K}}\left(\sigma_{1} \circ \sigma_{2}\right)=c_{\mathcal{A} / \mathcal{K}}\left(\sigma_{1}\right) \sigma_{1} \cdot c_{\mathcal{A} / \mathcal{K}}\left(\sigma_{2}\right),
$$

for $\sigma_{1}, \sigma_{2} \in \mathcal{D}$.

To understand why $c_{\mathcal{A} / \mathcal{K}}$ extends to the support of $\nu_{T}$, and why the extension is an SDiff-cocycle, we must have an explicit formula for it. Let $j$ denote the original complex structure, and let $j^{\prime}=\sigma \cdot j$ denote the transformed complex structure; we do the same for the corresponding D-bar operators, *-operators, and so on.

Lemma 2.14. Suppose that $\sigma \in \mathcal{D}$, and let $\mu=\mu_{\sigma^{-1}}$.

(a) $\left(\bar{\partial}^{\prime}\right)^{0,1}=(1-\mu \bar{\mu})^{-1}(\bar{\partial}-\mu \partial)$.

(b) $\left(\bar{\partial}^{\prime}\right)^{1,0}=\bar{\mu}\left(\bar{\partial}^{\prime}\right)^{0,1}$.

(c) $a^{\prime}=(1+\bar{\mu})(1-\mu \text { conj })^{-1} a$, where conj denotes conjugation.

(d) $x^{\prime}=(\bar{\partial}-\mu \partial)^{-1}(\bar{\partial}+\mu \partial) x$, for $\mathbb{R}$-valued $x ; x^{\prime}=x$, for $i \mathbb{R}$-valued $x$.

(e) $\partial^{\prime} \bar{\partial}^{\prime}=2 \partial \bar{\partial}\left(\frac{1+\bar{\partial}^{-1} \mu \partial}{1-\bar{\partial}^{-1} \mu \partial}+\frac{1+\partial^{-1} \bar{\mu} \bar{\partial}}{1-\partial^{-1} \bar{\mu} \bar{\partial}}\right)^{-1}$.

(f) $\sigma \cdot \operatorname{det}(\operatorname{det})^{*}=\exp \left(i \int\left(1-\bar{\partial}^{-1} \mu \partial\right)^{-1} x \wedge F\right)=\exp (-S(\mu) F \cdot F)$. where $S(\mu)=i *(\partial(\bar{\partial}-\mu \partial))^{-1}$.

(g) $c_{\mathcal{A} / \mathcal{K}}(\sigma, F)=\exp (-(S(\mu)-S(0)) F \cdot F)$.

(h) The real and imaginary parts of $S(\mu)$ are symmetric, with respect to the real Hilbert space structure (2.4). 
(i) $\mathbb{R e}(S(\mu))=i *\left(\partial^{\prime} \bar{\partial}^{\prime}\right)^{-1}=2 * *^{\prime} \Delta^{\prime-1}$ (the Laplacian on 2-forms); hence if $\sigma \in S \operatorname{Diff}, \mathbb{R e} S(\mu)=2 \Delta^{\prime-1}$.

Proof of (2.14). In $j$-holomorphic coordinates suppose that $w=\sigma(z), f=$ $f(w)$. Then

$$
\begin{aligned}
\bar{\partial}_{w}^{\prime} f & =\sigma^{-1 *}\left(\bar{\partial}_{z}\{f \circ \sigma\}\right) \\
& =\left(f_{\bar{w}} \bar{w}_{\bar{z}} \circ \sigma^{-1}+f_{w} w_{\bar{z}} \circ \sigma^{-1}\right) d \bar{z} \\
& =\left(f_{\bar{w}}-\mu f_{w}\right) \bar{w}_{\bar{z}} \circ \sigma^{-1} \bar{z}_{\bar{w}} d \bar{w}+\left(f_{\bar{w}}-\mu f_{w}\right) \bar{w}_{\bar{z}} \circ \sigma^{-1} \bar{z}_{w} d w .
\end{aligned}
$$

Using (1.17), we see that

$$
\bar{w}_{\bar{z}} \circ \sigma^{-1} \bar{z}_{\bar{w}}=\left(\frac{z_{w}}{z_{w} \bar{z}_{\bar{w}}-z_{\bar{w}} \bar{z}_{w}}\right) \bar{z}_{\bar{w}}=\frac{1}{1-\mu \bar{\mu}} .
$$

Similarly $\bar{w}_{\bar{z}} \circ \sigma^{-1} \bar{z}_{w}=\bar{\mu}(1-\mu \bar{\mu})^{-1}$. This proves (a) and (b).

We have $\bar{\partial} x=a, \bar{\partial}^{\prime} x^{\prime}=a^{\prime}$, where

$$
A=a-a^{*}=a^{\prime}-a^{*} .
$$

This implies

$$
\begin{aligned}
a & =\left(\bar{\partial}^{\prime} x^{\prime}\right)^{0,1}-\operatorname{conj}\left(\left(\bar{\partial}^{\prime} x^{\prime}\right)^{1,0}\right) \\
& =(1-\mu \bar{\mu})^{-1}(1-\mu \operatorname{conj})(\bar{\partial}-\mu \partial) x^{\prime} \\
& =(1+\mu \text { conj })^{-1}(\bar{\partial}-\mu \partial) x^{\prime} .
\end{aligned}
$$

Hence

$$
a^{\prime}=\bar{\partial}^{\prime}\left\{(\bar{\partial}-\mu \partial)^{-1}(1+\mu \text { conj }) a\right\}=(1+\bar{\mu})(1-\mu \text { conj })^{-1} a,
$$

which proves (c), and also

$$
(\bar{\partial}-\mu \partial) x^{\prime}=(1+\mu \text { conj }) \bar{\partial} x,
$$

which implies (d).

We have

$$
(\bar{\partial}-\mu \partial)^{-1}(\bar{\partial}+\mu \partial)=\frac{1+\bar{\partial}^{-1} \mu \partial}{1-\bar{\partial}^{-1} \mu \partial} .
$$

For $\mathbb{R}$-valued $x$

$$
2 \partial \bar{\partial} x=F=2 \partial^{\prime} \bar{\partial}^{\prime} \mathbb{R} e\left(x^{\prime}\right),
$$

hence (e) follows from (2.20) and (d).

For $\mathbb{R}$-valued $x$,

$$
\sigma \cdot \operatorname{det}(\operatorname{det})^{*}=e^{i \int\left(x^{\prime}+x\right) \wedge F}=e^{i \int \frac{2}{1-\bar{\partial}^{-1} \mu \partial} x \wedge F},
$$

which is (f). Part (g) follows immediately from (f).

In part (h) it suffices to show that $(\partial \bar{\partial}-\partial \mu \partial) *$ is symmetric, and this is immediate from integration by parts. 
We have $S(0)=2 \Delta$ (acting on 2 -forms), and by (f) $|\operatorname{det}|=\exp (-2 \Delta F \cdot F)$. Together with $(\mathrm{g})$ we now see that

$$
\begin{aligned}
|\sigma \cdot \operatorname{det}| & =\exp \left(-2 \Delta^{\prime} F \cdot^{\prime} F\right) \\
& =\exp \left(-2 * *^{\prime} \Delta^{\prime} F \cdot F\right)=\exp (-\mathbb{R} e S(\mu) F \cdot F) .
\end{aligned}
$$

This proves (i).

Given a real Hilbert space $\mathcal{H}$ and a symmetric Hilbert-Schmidt operator $S$ on $\mathcal{H}$ (possibly with values in $\mathcal{H}^{\mathbb{C}}$ ), one can interpret "Sv$\cdot v-\operatorname{tr} S$ " as a random variable with respect to the Gaussian measure $d \nu$ associated to $\mathcal{H}$. More precisely, if $\left\{\epsilon_{n}\right\}$ is an orthonormal basis for $\mathcal{H}$ (hence also $\mathcal{H}^{\mathbb{C}}$ ), and $P_{n}$ the orthogonal projection onto the $\mathbb{C}$-span of the first $n$ basis elements, then the regularization of the symmetric form defined by $S$ is the $L^{2}(d \nu)$ limit

$$
S_{\text {reg }}=\lim _{n \rightarrow \infty}\left(P_{n} S x \cdot x-\operatorname{tr}\left(P_{n} S\right)\right) .
$$

This definition is independent of the choice of orthonormal basis. Moreover

$$
\int e^{-S_{\mathrm{reg}}} d \nu=\operatorname{det}\left((1+2 S) e^{-2 S}\right)^{-1 / 2},
$$

and if $S^{\prime}$ is also Hilbert-Schmidt and symmetric, and $O \in O(H)$, then

$$
\begin{aligned}
& \left(S+S^{\prime}\right)_{\mathrm{reg}}=S_{\mathrm{reg}}+S_{\mathrm{reg}}^{\prime}, \\
& O \cdot\left(S_{\mathrm{reg}}\right)=\left(O S O^{-1}\right)_{\mathrm{reg}}
\end{aligned}
$$

(see Section 4 of $[\mathbf{R}]$ ).

In our context the Hilbert-Schmidt operator is $\Delta^{-1}$, or some $0^{\text {th }}$-order perturbation. (Note that $\Delta^{-1}$, while not trace class, is in the Dixmier trace class $\mathcal{L}_{1}^{+}$, so that the regularization in our case is necessary, but very mild.)

Definition 2.27. Given $\sigma \in \mathcal{D}$,

$$
c_{T}(\sigma)=\exp \left(-T(S(\mu)-S(0))_{\mathrm{reg}}\right)
$$

(a random variable with respect to $\nu_{T}$ ).

Remarks 2.28. (a) The appearance of $T$ in the above definition is potentially confusing; it appears because we are regularizing with respect to $\nu_{T}$, the Gaussian corresponding to the inner product $\frac{1}{T} F \cdot F$.

(b) In the next section we will see that (2.27) is fine for $\sigma \in S$ Diff, but needs adjustment for general $\mu$; see Remark (3.30) below, expecially (3.32). The point is that one cannot indiscrimately subtract a trace!

Proposition 2.29. $c_{T}$ is an SDiff-cocycle with respect to $\nu_{T}$, i.e.,

$$
c_{T}\left(\sigma_{1} \circ \sigma_{2}\right)=c_{T}\left(\sigma_{1}\right) \sigma_{1} \cdot c_{T}\left(\sigma_{2}\right)
$$

as random variables with respect to $\nu_{T}, \forall \sigma_{1}, \sigma_{2} \in S$ Diff. 
Proof. This follows immediately from (2.13) and (2.26), since SDiff acts orthogonally with respect to $\nu_{T}$.

Although it is somewhat artificial in our current context, we can now view $\mathcal{L}$ as a measureable line bundle over the support of $\nu_{T}$, using the transition functions $c_{T}(\sigma), \sigma \in S$ Diff.

Definition 2.30. We define a measure $d \nu_{T}^{|\mathcal{L}|^{-2}}$ having values in the line bundle $|\mathcal{L}|^{-2}$ by

$$
s_{1} \bar{s}_{2} d \nu_{T}^{|\mathcal{L}|^{-2}}=\frac{s_{1}}{\operatorname{det}}\left(\frac{s_{2}}{\operatorname{det}}\right)^{*} e^{-T S(0)_{\mathrm{reg}}} d \nu_{T},
$$

where $s_{1}, s_{2}$ are sections of $\mathcal{L}$ over the support of $\nu_{T}$.

Proposition 2.31. (a) $\frac{d \sigma_{*}|\operatorname{det}|^{2} d \nu_{T}^{|\mathcal{L}|^{-2}}}{d|\operatorname{det}|^{2} d \nu_{T}^{|\mathcal{L}|^{-2}}}=\left|c_{T}(\sigma)\right|^{2}$.

(b) $d \nu_{T}^{|\mathcal{L}|^{-2}}$ is SDiff-invariant.

Proof of (2.31). Part (a) follows immediately from the definition of the measure

$$
|\operatorname{det}|^{2} d \nu_{T}^{|\mathcal{L}|^{-2}}=e^{-T S(0)_{\mathrm{reg}}} d \nu_{T}
$$

and the fact that $\sigma_{*}\left(S(0)_{\mathrm{reg}}\right)=\mathbb{R e}\left(S(\mu)_{\mathrm{reg}}\right)$, by (i) of $(2.14)$.

Part (b) is a restatement of (a).

We can now define a $S$ Diff-invariant unitary structure on sections of $\mathcal{L}$ over the support of $\nu_{T}$ by

$$
\left\langle s_{1}, s_{2}\right\rangle=\int s_{1} \bar{s}_{2} d \nu_{T}^{|\mathcal{L}|^{-2}} .
$$

We let $\pi_{T}$ denote the unitary representation of SDiff acting on sections of $\mathcal{L}$ over the support of $\nu_{T}$. We can equivalently view $\pi_{T}$ as the cocycle representation

$$
S \operatorname{Diff} \times L^{2}\left(|\operatorname{det}|^{2} d \nu_{T}^{|\mathcal{L}|^{-2}}\right)
$$

given by $\sigma \cdot f=\left(f \circ \sigma^{-1}\right) c_{T}(\sigma)$ (where the function $f$ corresponds to the section $s=f$ det).

Proposition 2.35. The spherical function for the representation $\pi_{T}$ of SDiff corresponding to the vector det is given by

$$
\begin{aligned}
\left\langle\pi_{T}(\sigma) \cdot \operatorname{det}, \operatorname{det}\right\rangle & =\int \sigma \cdot \operatorname{det}(\operatorname{det})^{*} d \nu_{T}^{|\mathcal{L}|^{-2}} \\
& \left.=\int e^{-T S(\mu)_{\mathrm{reg}}} d \nu_{T}=\operatorname{det}(1+2 T S(\mu)) e^{-2 T S(\mu)}\right)^{-1 / 2} .
\end{aligned}
$$

This follows from (2.25). 
Remarks 2.36. (a) There is a simple explanation for why the vector det is not a smooth vector for the unitary action of $S$ Diff. Given $v \in \operatorname{Vect}(\Sigma)$,

$$
\begin{aligned}
\left.v \cdot \operatorname{det}\right|_{A} & =\left.\left.\frac{d}{d t}\right|_{t=0} e^{t v} \cdot \operatorname{det}\right|_{A}=\left.\operatorname{det}\right|_{A}\left(\left.d(\log \operatorname{det})\right|_{A}\left(-\mathcal{L}_{v} A\right)\right) \\
& =\left.i \operatorname{det}\right|_{A}\left(\int\left(i_{v}\left\{\bar{\partial}^{-1} F\right\}-\bar{\partial}^{-1}\left\{\left(i_{v} F\right)^{0,1}\right\}\right) \wedge F\right) .
\end{aligned}
$$

The operator

$$
F \rightarrow *\left(i_{v}\left\{\bar{\partial}^{-1} F\right\}-\bar{\partial}^{-1}\left\{\left(i_{v} F\right)^{0,1}\right\}\right)
$$

has order $=-1$, hence generally it is not Hilbert-Schmidt, and it is not possible to regularize the corresponding quadratic form.

(b) The partition function of abelian Yang-Mills (at temperature T) coupled to fermions (with coupling constant 1 ) is the integral

$$
\mathcal{Z}(T)=\int|\operatorname{det}|^{2} d \nu_{T}^{|\mathcal{L}|^{-2}}=\left(\operatorname{det}\left(1+4 T \Delta^{-1}\right) e^{-4 T \Delta^{-1}}\right)^{-1 / 2} .
$$

An interesting question is whether one can compute the corresponding quantity in the nonabelian case.

\section{The limit $T \rightarrow \infty$.}

As in the preceding section, $\Sigma$ denotes a genus $=0$ oriented surface, and we consider the representation (2.2). We fix a complex structure, so that we have a distinguished section det. We also fix an area form, so that we can use the Yang-Mills construction of the preceding section. Our objective is to prove that the $S$ Diff-submodule generated by the section det is unitary with respect to the Hermitian inner product

$$
\left\langle\sigma_{1} \cdot \operatorname{det}, \sigma_{2} \cdot \operatorname{det}\right\rangle=\lim _{T \rightarrow \infty} \frac{1}{\mathcal{Z}(T)} \int c_{T}\left(\sigma_{2}^{-1} \sigma_{1}\right)|\operatorname{det}|^{2} d \nu_{T}^{|\mathcal{L}|^{-2}}
$$

where $\mathcal{Z}(T)$ is the partition function in (b) of (2.36).

Proposition 3.2. Suppose that $\sigma \in S$ Diff, $\mu=\mu_{\sigma^{-1}}$. Then

$$
\lim _{T \rightarrow \infty} \frac{\operatorname{det}\left((1+2 T S(\mu)) e^{-2 T S(\mu)}\right)^{-1 / 2}}{\operatorname{det}\left((1+2 T S(0)) e^{-2 T S(0)}\right)^{-1 / 2}}=\frac{\operatorname{det}_{\zeta}(4 \Delta)^{1 / 2}}{\operatorname{det}_{\zeta}\left(4 \Delta\left(1-\bar{\partial}^{-1} \mu \partial\right)^{-1}\right)^{1 / 2}},
$$

and this defines a positive definite function on SDiff.

To explain the meaning of the RHS of (3.2), and before undertaking the proof, we need some preparatory remarks concerning determinants (we will use $[\mathbf{S h}]$ and $[\mathbf{K V}]$ as basic references). Suppose that $E$ is an invertible elliptic classical pseudo-differential operator of positive real order $d$, on a compact manifold of dimension $n$, and suppose that its principal symbol $\sigma_{d}$ satisfies the Agmon-Nirenberg condition, i.e., there is a ray $L_{\theta}$ extending from the origin in the complex plane such that at each point of the manifold, 
the spectrum of $\sigma_{d}$ intersects trivially with $L_{\theta}$. In our context $E$ will always have the property that we can choose $L_{\theta}$ to be the negative real axis, as in $\S 10$ of $[\mathbf{S h}]$. In this situation one can form the complex powers $E^{-s}, s \in \mathbb{C}$, and the $\zeta$-function

$$
\zeta_{E}(s)=\operatorname{tr}\left(E^{-s}\right), \quad \mathbb{R} e(s)>n / d,
$$

has a meromorphic extension to all of $\mathbb{C}$ which is regular in a neighborhood of $s=0$ ( $\$ 10$ and $\S 13$ of $[\mathbf{S h}]$, respectively). We then define the $\zeta$ determinant in the usual way, $\operatorname{det}_{\zeta} E=\exp \left(-\zeta^{\prime}(0)\right)$. In particular this is the meaning of the determinants on the RHS of (3.2), where we have thrown out the zero eigenvalue (note that the symbol of $\Delta\left(1-\bar{\partial}^{-1} \mu \partial\right)^{-1}$ is $\rho|\xi|^{2}\left(1-\tilde{\mu}(z) \bar{\xi}^{-1} \xi\right)^{-1}$, where $\tilde{\mu}$ and $\rho$ are local representations for $\mu$ and the area form, respectively; since $|\tilde{\mu}|<1$, the negative real axis is a spectral cut).

Now initially suppose also that $C$ is a pseudo-differential operator of order $<-n$, so that $C$ represents a trace class operator. In this case we have

$$
\operatorname{det}_{\zeta}\left(E e^{\tau C}\right)=\left(\operatorname{det}_{\zeta} E\right) e^{\tau \operatorname{tr} C} .
$$

To see this, note that $\left(E e^{\tau C}\right)^{-s} C$ is a trace class operator for all $s \in \mathbb{C}$, hence the trace of this holomorphic family is an entire function of $s$. Also

$$
\frac{\partial}{\partial \tau} \zeta_{E e^{\tau C}}(s)=\frac{\partial}{\partial \tau} \operatorname{tr}\left(E e^{\tau C}\right)^{-s}=-s \operatorname{tr}\left(\left(E e^{\tau C}\right)^{-s} C\right), \quad \mathbb{R} e(s)>n / d
$$

By analytic continuation this equality is valid in all of $\mathbb{C}$, in particular in a neighborhood of $s=0$. Hence

$$
\frac{\partial}{\partial \tau} \zeta_{E e^{\tau C}}^{\prime}(0)=-\operatorname{tr} C
$$

implying that

$$
\frac{\partial}{\partial \tau} \operatorname{det}_{\zeta}\left(E e^{\tau C}\right)=\operatorname{det}_{\zeta}\left(E e^{\tau C}\right) \operatorname{tr} C
$$

which integrates to (3.4).

Now suppose that order $(C) \leq-n$. In this case $C \in \mathcal{L}_{1}^{+}$(the dual of the Macaev ideal; see $[\mathbf{C}])$, and $\left(E e^{\tau C}\right)^{-s} C$ is trace class for $\mathbb{R} e(s)>0$. The trace of this family has a meromorphic extension to the complex plane, and in a neighborhood of $s=0$, we have

$$
\operatorname{tr}\left(\left(E e^{\tau C}\right)^{-s} C\right)=\frac{\operatorname{Res}(C)}{s}+h(s),
$$

where $\operatorname{Res}(C)$ is the noncommutative residue and $h(s)$ is holomorphic. The value of $h$ at $s=0$ is called the finite part of the trace; we will write " $\mathcal{F} \mathcal{P}$ tr" for the finite part:

$$
\mathcal{F} \mathcal{P} \operatorname{tr}\left(\left(E e^{\tau C}\right)^{-s} C\right)=h(0) .
$$

Note that if order $(C)<-n$, then $\operatorname{Res}(C)=0$ and $h(0)=\operatorname{tr} C$. 
Lemma 3.10. Suppose that $E$ is classical, elliptic, invertible and satisfies the Agmon-Nirenberg condition as above, and $C$ has order $\leq-n$. Then

(a) $\operatorname{det}_{\zeta}\left(E e^{\tau C}\right)=\operatorname{det}_{\zeta}(E) e^{\tau \mathcal{F} \mathcal{P} \operatorname{tr}\left(E^{-s} C\right)}$.

(b) The linear functional $C \rightarrow \mathcal{F P} \operatorname{tr}\left(E^{-s} C\right)$ depends only upon the principal symbol of $E$.

Proof of (3.10). We have

$$
\begin{aligned}
\mathcal{F} \mathcal{P} \operatorname{tr}\left(E e^{\tau C}\right)^{-s} C= & \mathcal{F} \mathcal{P} \operatorname{tr}\left(\left(E e^{\tau C}\right)^{-s} C-E^{-s} e^{-s \tau C} C\right) \\
& +\mathcal{F} \mathcal{P} \operatorname{tr}\left(E^{-s} e^{-s \tau C} C-E^{-s} C\right)+\mathcal{F} \mathcal{P} \operatorname{tr}\left(E^{-s} C\right) .
\end{aligned}
$$

We must show that the first two terms on the RHS are zero. As $s \rightarrow 0$,

$$
\left(E e^{\tau C}\right)^{-s} C-E^{-s} e^{-s \tau C} C=-s\left(\log \left(E e^{\tau C}\right)-\log E-\tau C\right) C+O\left(s^{2}\right)
$$

where we have used a spectral cut near $L_{\theta}$ to form the logarithms (see (2.8) of $[\mathbf{K V}]$ for the definition of the logarithms and basic properties). The (non-classical) pseudo-differential operator $\log \left(E e^{\tau C}\right)-\log E-\tau C$ has order $\leq-1+\epsilon$, for any $\epsilon>0$, because the log and 0th order terms cancel out in the calculation of the symbol, and we are assuming that $E$ is classical. Thus the RHSs of both (3.12) and (3.13) are of the form $-s T+O\left(s^{2}\right)$, where $T$ is trace class. It follows that the first two terms on the RHS of (3.11) are zero.

As before

$$
\frac{\partial}{\partial \tau} \zeta_{E e^{\tau C}}(s)=-\operatorname{str}\left(\left(E e^{\tau C}\right)^{-s} C\right), \quad \mathbb{R} e(s)>0 .
$$

Since the first two terms on the RHS of (3.12) are zero, as $s \rightarrow 0$,

$$
\frac{\partial}{\partial \tau} \zeta_{E e^{\tau C}}(s)=-\mathcal{F} \mathcal{P} \operatorname{tr}\left(E^{-s} C\right)+O(s) .
$$

Thus

$$
\frac{\partial}{\partial \tau} \operatorname{det}_{\zeta}\left(E e^{\tau C}\right)=\operatorname{det}_{\zeta}\left(E e^{\tau C}\right) \mathcal{F} \mathcal{P} \operatorname{tr}\left(E^{-s} C\right),
$$

and this completes the proof of (a)

The proof of (b) follows the same pattern as for (a). Suppose that order $(F)<\operatorname{order}(E)$, where $E+F$ is also invertible. Then we can write $E+F=E(1+B)$, where order $(B) \leq-1$, and since the spectrum of $1+B$ is discrete, we can find a spectral cut to form the compex powers of $1+B$. Then

$$
\mathcal{F} \mathcal{P} \operatorname{tr}\left((E(1+B))^{-s} C\right)=\mathcal{F} \mathcal{P} \operatorname{tr}\left(E^{-s}(1+B)^{-s} C\right)=\mathcal{F} \mathcal{P} \operatorname{tr}\left(E^{-s} C\right),
$$

where the first equality uses $(3.12)$ with $\log (1+B)$ (which is of order $\leq-1$ ) in place of $C$, and the second uses (3.13) in the same way. 
Proof of (3.2). In the preceding section we viewed $S(\mu)$ as an operator on a Hilbert space of two-forms. In this proof we will replace $S(\mu)$ by $* S(\mu) *$, so that we can view it as an operator on functions. Also recall that $\Delta^{\prime}=$ $\sigma \circ \Delta \circ \sigma^{-1}$.

Fix $T>0$. Let $E$ denote an operator as in (3.10) (for us $E=\Delta$ or $\Delta^{\prime}$ ). The manifold $\Sigma$ has dimension 2, the operator $S(\mu)$ has order -2 , and the operator $(1+2 T S(\mu)) e^{-2 T S(\mu)}$ is of the form $1+\operatorname{order}(-4)$. Hence by $(3.4)$ and (3.10),

$$
\begin{aligned}
& \operatorname{det}_{\zeta}\left(\frac{1}{T} E^{2}\right) \operatorname{det}\left((1+2 T S(\mu)) e^{-2 T S(\mu)}\right) \\
& =\operatorname{det}_{\zeta}\left(E(T, \mu) e^{-2 T S(\mu)}\right) \\
& =\operatorname{det}_{\zeta}(E(T, \mu)) \exp \left(-2 T \mathcal{F P} \operatorname{tr}\left(E(T, \mu)^{-s} S(\mu)\right)\right),
\end{aligned}
$$

where $E(T, \mu)=\frac{1}{T} E^{2}(1+2 T S(\mu))$.

Now we claim that

$$
\begin{aligned}
\mathcal{F} \mathcal{P} \operatorname{tr}\left(E(T, \mu)^{-s} S(\mu)\right) & =\mathcal{F} \mathcal{P} \operatorname{tr}\left(\left(\frac{1}{T} E^{2}\right)^{-s}(1+2 T S(\mu))^{-s} S(\mu)\right) \\
& =\mathcal{F} \mathcal{P} \operatorname{tr}\left(\left(\frac{1}{T} E^{2}\right)^{-s} S(\mu)\right) \\
& =\operatorname{Re} s(S(\mu)) \ln T+\mathcal{F} \mathcal{P} \operatorname{tr}\left(E^{-s} S(\mu)\right) .
\end{aligned}
$$

The first two equalities follow from (b) of (3.10). The third equality follows simply from $T^{s}=1+\sin T+O\left(s^{2}\right)$,

$$
\operatorname{tr}\left(E^{-s} S(\mu)\right)=\frac{\operatorname{Re}(S(\mu))}{s}+\mathcal{F} \mathcal{P} \operatorname{tr}\left(E^{-s} S(\mu)\right)+O(s) \quad \text { as } \quad s \rightarrow 0
$$

and the fact that changing the argument in a meromorphic function from $s$ to $2 s$ does not change the constant term in a Laurent expansion.

From (3.18) and (3.19) we therefore conclude that

$$
\begin{aligned}
& \operatorname{det}_{\zeta}\left(\frac{1}{T} E^{2}\right) \operatorname{det}\left((1+2 T S(\mu)) e^{-2 T S(\mu)}\right) \\
& =\operatorname{det}_{\zeta}\left(\frac{1}{T} E^{2}+2 E^{2} S(\mu)\right) T^{-T \operatorname{Re} s(S(\mu))} e^{-T \mathcal{F P} \operatorname{tr}\left(E^{-s} 2 S(\mu)\right)} .
\end{aligned}
$$

Since $\Delta$ and $\Delta^{\prime}$ have the same spectrum, (3.21) implies that

$$
\frac{\operatorname{det}_{\zeta}\left(\frac{1}{T} \Delta^{2}+\Delta^{2} S(\mu)\right)}{\operatorname{det}_{\zeta}\left(\frac{1}{T} \Delta^{\prime 2}+\Delta^{\prime 2} S(\mu)\right)}=\exp \left(-T \mathcal{F} \mathcal{P} \operatorname{tr}\left(\left(\Delta^{-s}-\Delta^{\prime-s}\right) S(\mu)\right)\right) .
$$

Since the LHS of (3.22) has a finite limit as $T \rightarrow \infty$, we conclude that

$$
\mathcal{F} \mathcal{P} \operatorname{tr}\left(\Delta^{-s} S(\mu)\right)=\mathcal{F} \mathcal{P} \operatorname{tr}\left(\Delta^{\prime-s} S(\mu)\right) .
$$


Let $C(\sigma)=S(\mu)-S(0)$, where $\mu=\mu_{\sigma^{-1}}$. Using (g) of (2.14), we see that (2.13) translates into the equality of operators

$$
C\left(\sigma_{1} \circ \sigma_{2}\right)=C\left(\sigma_{1}\right)+\sigma_{1} C\left(\sigma_{2}\right) \sigma_{1}^{-1},
$$

for $\sigma_{1}, \sigma_{2} \in S$ Diff. It follows from (3.23) and (3.24) that

$$
S \text { Diff } \rightarrow \mathbb{C}: \sigma \rightarrow \mathcal{F} \mathcal{P} \operatorname{tr}\left(\Delta^{-s} C(\sigma)\right)
$$

is a homomorphism of groups. But the only such homomorphism of groups is the trivial map. We therefore conclude that

$$
\mathcal{F} \operatorname{Ptr}\left(\Delta^{-s}(S(\mu)-S(0))\right)=0,
$$

provided that $\mu$ corresponds to a $\sigma \in S$ Diff. [Note: There is a more direct way to see that the real part of (3.26) is zero, for by (i) of (2.14) and (3.23),

$$
\left.\mathbb{R} e \mathcal{F} \mathcal{P} \operatorname{tr}\left(\Delta^{-s}(S(\mu)-S(0))\right)=2 \mathcal{F} \mathcal{P} \operatorname{tr}\left(\Delta^{\prime-s} \Delta^{\prime-1}-\Delta^{-s} \Delta^{-1}\right)=0 .\right]
$$

Now the principal symbol of $S(\mu)$ is of the form

$$
\rho|\xi|^{-2}(1-\xi \tilde{\mu} \xi)^{-1}
$$

where $\rho$ and $\tilde{\mu}$ are the local representations for $\mu$ and our fixed area element, respectively. Since the density for $\operatorname{Re} s(S(\mu))$ can be computed by integrating over $|\xi|=1$, it follows that $\operatorname{Re} s(S(\mu))$ is independent of $\mu$. Now combining this with (3.21) we have

$$
\begin{aligned}
& \frac{\operatorname{det}\left((1+2 T S(\mu)) e^{-2 T S(\mu)}\right)}{\operatorname{det}\left((1+2 T S(0)) e^{-2 T S(0)}\right)} \\
& =\frac{\operatorname{det}_{\zeta}\left(\frac{1}{T} \Delta^{2}+\Delta^{2} 2 S(\mu)\right)}{\operatorname{det}_{\zeta}\left(\frac{1}{T} \Delta^{2}+4 \Delta\right)} e^{-2 T \mathcal{F P} \operatorname{tr}\left(\Delta^{-s} S(\mu)-\Delta^{-s} S(0)\right)}
\end{aligned}
$$

for any $\mu$. By (3.26), if $\mu$ corresponds to $\sigma \in S$ Diff, then the exponential term vanishes. One can now take the limit $T \rightarrow \infty$. This completes the proof.

Remarks 3.30. It is very unlikely that (3.26) vanishes for a general $\mu$, hence the limit in (3.2) probably does not exist for general $\mu$. But this makes perfectly good sense for the following reason. In the previous section, following standard practice in probability theory, we regularized

$$
\sigma \cdot \operatorname{det}(\operatorname{det})^{*}=\exp (-S(\mu) F \cdot F)
$$

by subtracting a trace, that is we replaced $S(\mu) F \cdot F$ by " $S(\mu) F \cdot F-\operatorname{tr} S(\mu)$ ", where this had to be properly interpreted as a random variable with respect to $\nu_{T}$. The proof of (3.2) makes it clear that this is inadequate; we should actually have replaced (3.31) by

$$
\exp \left(-T \mathcal{F} \mathcal{P} \operatorname{tr}\left(\Delta^{-s} S(\mu)\right)\right) \exp \left(-T S(\mu)_{\operatorname{reg}\left(\nu_{T}\right)}\right),
$$

that is, we should have added the trace back in! If we make this change, then the limit in (3.2) exists for all $\mu$. 


\section{The Cases genus $(\Sigma)>0$.}

We briefly indicate the changes necessary to handle the case in which genus $(\Sigma)$ is positive.

First, it is no longer the case that the gauge group is connected; in fact, we can identify

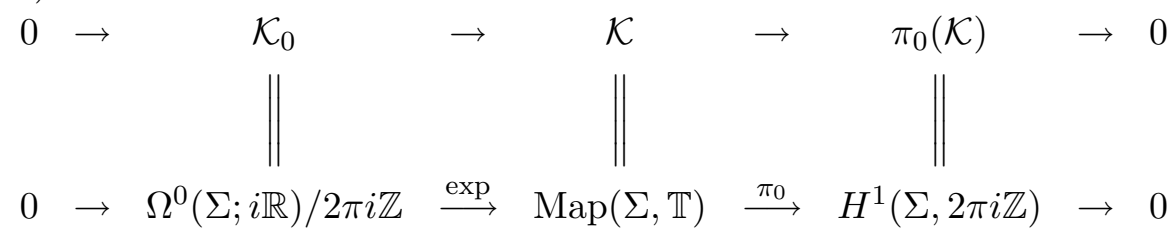

where $\pi_{0}(g)=\left[g^{-1} d g\right] \in H^{1}(\Sigma, 2 \pi i \mathbb{Z})$. We define

$$
\Omega^{0}(\mathcal{L})=\left\{f: \mathcal{A} \rightarrow \mathbb{C}: f\left(A+g^{-1} d g\right)=\mathbf{c}(g, A) f(A), \quad \forall A \in \mathcal{A}, g \in \mathcal{K}\right\}
$$

where $\mathbf{c}(g, A)=\exp \left(\frac{1}{2 \pi i} \int A \wedge g^{-1} d g\right)$. The group $\mathcal{D}$ acts naturally on $\Omega^{0}(\mathcal{L})$.

Remarks 4.3. (a) $\Omega^{0}(\mathcal{L})$ is the space of sections of a $\mathcal{D}$-equivariant line bundle over $\mathcal{C}$, i.e., c satisfies the cocycle identity

$$
\mathbf{c}\left(g_{1} g_{2}, A\right)=\mathbf{c}\left(g_{1}, A\right) \mathbf{c}\left(g_{2}, A+g_{1}^{-1} d g_{1}\right) ;
$$

this depends crucially on the fact that

$$
\exp \left(\frac{1}{2 \pi i} \int g_{2}^{-1} d g_{2} \wedge g_{1}^{-1} d g_{1}\right)=1
$$

(b) There is a refinement of (4.2), which depends upon the additional choice of a spin structure on $\Sigma$. A spin structure determines a function

$$
(-1)^{q}: H^{1}(\Sigma, 2 \pi i \mathbb{Z}) \rightarrow\{ \pm 1\}
$$

satisfying $(-1)^{q\left(\lambda_{1}+\lambda_{2}\right)}=(-1)^{q\left(\lambda_{1}\right)}(-1)^{q\left(\lambda_{2}\right)} e^{\frac{1}{4 \pi i} \int \lambda_{1} \wedge \lambda_{2}}$, where $q$ of a simple loop is 0 or 1 , depending upon whether the spin structure restricted to the loop is trivial or nontrivial. We then define $\Omega^{0}\left(\mathcal{L}_{q}\right)$ to consist of functions satisfying

$$
f\left(A+g^{-1} d g\right)=(-1)^{q\left(\pi_{0}(g)\right)} \exp \left(\frac{1}{4 \pi i} \int A \wedge g^{-1} d g\right) f(A) .
$$

The $(-1)^{q}$ factor compensates for the fact that the square root of the LHS of (4.5) may not be 1 . In this case we have a representation of $\mathcal{D}_{q}$ on $\Omega^{0}\left(\mathcal{L}_{q}\right)$.

To define the Yang-Mills measure, we must fix an area form as before. As in the genus $=0$ case, the support of the Yang-Mills measure is thicker than the space of gauge equivalence classes of continuous $\mathbb{T}$-connections, $\mathcal{C}$. To complete the space $\mathcal{C}$ in a $\mathcal{D}$-equivariant way, one can adopt the point of view that a connection is a parallel transport functor, hence that $\mathcal{C}$ consists 
of holonomy functors. This is described in $[\mathbf{P i}]$, see especially $\S 2.5$ for the abelian case. The resulting completion of $\mathcal{C}$ has the structure of a principal bundle

$$
\begin{gathered}
\mathcal{C}^{\text {completion }} \\
\left\{F \in \Omega^{2}: \int F=0\right\}^{\text {completion }}
\end{gathered}
$$

over a completion of the space of curvatures, where

$$
H^{1}(\Sigma, \mathbb{T})=\operatorname{Hom}\left(\pi_{1}(\Sigma), \mathbb{T}\right) .
$$

The Yang-Mills measure, again denoted by $d \nu_{T}$, is the unique $H^{1}(\Sigma, \mathbb{T})$ invariant probability measure on the completion of $\mathcal{C}$ which projects to the Gaussian corresponding to the inner product (2.4).

Now fix a complex structure for $\Sigma$. The section det is characterized in the following:

Lemma 4.10. There is a unique section $\operatorname{det}$ of $\mathcal{L}$ with $\operatorname{det}(0)=1$ such that

$$
\exp \left(\frac{1}{2 \pi i} \int a \wedge a^{*}\right) \operatorname{det}
$$

is a holomorphic function of a.

Proof. There is a canonical splitting of the sequence

$$
\begin{gathered}
0 \rightarrow \Omega^{0} / \mathbb{C} \rightarrow \Omega^{0,1} \rightarrow H^{0,1} \rightarrow 0 \\
\Omega^{0,1}=\bar{\partial} \Omega^{0} \oplus \operatorname{ker}(\partial), \quad a=\bar{\partial} x+a_{0}
\end{gathered}
$$

because $\partial a=\partial \bar{\partial} x$ uniquely determines $x \in \Omega^{0} / \mathbb{C}$. Note that $x$ depends holomorphically on $a$. The function

$$
\exp \left(\frac{i}{2 \pi} \int\left\{a \wedge a^{*}-\partial a \wedge(\partial \bar{\partial})^{-1} \partial a\right\}\right)=\exp \left(\frac{i}{2 \pi} \int\left(a_{0} \wedge a_{0}^{*}+x \wedge F\right)\right)
$$

satisfies the transformation property (4.2) for $g=\exp (\xi) \in \mathcal{K}^{0}$, the identity component of $\mathcal{K}$. It follows that the section det has the form

$$
\operatorname{det}(A)=\exp \left(\frac{i}{2 \pi} \int\left(a_{0} \wedge a_{0}^{*}+x \wedge F\right)\right) \Theta(a),
$$

where $\Theta$ is a holomorphic function which is $\mathcal{K}^{0}$-invariant, hence $\mathcal{G}^{0}$-invariant, where $\mathcal{G}$ is the complexification of $\mathcal{K}$. Thus $\Theta$ descends to a function on $H^{0,1}$. For $g \in \mathcal{K}$, not necessarily in the identity component, as in (4.12) we can write uniquely

$$
g^{-1} d g=d \xi+\theta, \quad g^{-1} \bar{\partial} g=\bar{\partial} \xi+\theta^{0,1}
$$


where $\partial \theta^{0,1}=0, \xi \in \Omega^{0}(\Sigma, i \mathbb{R}) / i \mathbb{R}, \theta \in H^{1}(\Sigma, 2 \pi i \mathbb{Z})$. Then

$$
\begin{aligned}
& \Theta\left(a+g^{-1} \bar{\partial} g\right) \\
& =\frac{\operatorname{det}\left(A+g^{-1} d g\right)}{\exp \left(\frac{i}{2 \pi} \int\left\{\left(a+g^{-1} \bar{\partial} g\right) \wedge\left(a+g^{-1} \bar{\partial} g\right)^{*}-\partial\left(a+g^{-1} \bar{\partial} g\right) \wedge x\left(a+g^{-1} \bar{\partial} g\right)\right\}\right)} \\
& =\exp \left(\frac{i}{2 \pi} \int\left\{A \wedge g^{-1} d g-\left\{-a \wedge \theta^{1,0}+\theta^{0,1} \wedge\left(a^{*}-\theta^{1,0}\right)\right\}\right\}\right) \Theta(a) \\
& =\exp \left(\frac{i}{2 \pi} \int\left\{a \wedge 2 \theta^{0,1}-\theta^{0,1} \wedge \theta^{1,0}\right\}\right) \Theta(a) .
\end{aligned}
$$

Thus $\Theta: H^{0,1} \rightarrow \mathbb{C}$ satisfies

$$
\Theta\left(a+\lambda^{0,1}\right)=\exp \left(\frac{i}{2 \pi} \int\left(a \wedge 2 \lambda^{1,0}-\lambda^{0,1} \wedge \lambda^{1,0}\right)\right) \Theta(a)
$$

for all $a \in H^{0,1}, \lambda \in H^{1}(\Sigma, 2 \pi i \mathbb{Z})$. This implies that $\Theta$ is essentially Riemann's theta function

$$
\Theta(a)=\frac{1}{\mathcal{Z}} \sum_{\lambda} \exp \left(\frac{i}{2 \pi} \int \lambda^{1,0} \wedge\left(a+2 \lambda^{0,1}\right)\right) .
$$

Remark 4.19. If we choose a spin structure $q$ as in (b) of (4.3), i.e., a square root of the canonical bundle, then we can identify $\mathcal{L}_{q}$ with the determinant line of the family of Fredholm operators $\left\{\bar{\partial}_{\kappa^{1 / 2}}+a: a \in \Omega^{0,1}\right\}$, equipped with the Quillen metric. In this case the section det is identified with the canonical section, and (4.18) is replaced by the normalized theta function

$$
\Theta_{q}(a)=\frac{1}{\mathcal{Z}} \sum(-1)^{q} \exp \left(\frac{i}{4 \pi} \int \lambda^{1,0} \wedge\left(a+2 \lambda^{0,1}\right)\right) .
$$

Now suppose that $\sigma \in \mathcal{D}$. As in (2.14) we denote transformed objects using primes.

\section{Lemma 4.21.}

(a) $\left(\bar{\partial}^{\prime}\right)^{0,1}=(1-\mu \bar{\mu})^{-1}(\bar{\partial}-\mu \partial)$.

(b) $\left(\bar{\partial}^{\prime}\right)^{1,0}=\bar{\mu}\left(\bar{\partial}^{\prime}\right)^{0,1}$.

(c) $a^{\prime}=(1+\bar{\mu})(1-\mu \text { conj })^{-1} a$, where conj denotes conjugation.

(c) $a_{0}^{\prime}=f\left(\mu, a_{0}\right)$, where $f$ depends linearly on $a_{0}$.

(d) $x^{\prime}=(\partial \bar{\partial}-\partial \mu \partial)^{-1}\left\{(\partial \bar{\partial}+\partial \mu \partial) x+\partial\left[\mu \bar{a}_{0}+A_{0}^{\prime 0,1}-\mu A_{0}^{\prime 1,0}\right]\right\}$, for $\mathbb{R}$-valued $x$, where $A_{0}=a_{0}-\bar{a}_{0}$.

(e) $\partial^{\prime} \bar{\partial}^{\prime}=2 \partial \bar{\partial}\left((\partial \bar{\partial}-\partial \mu \partial)^{-1}(\partial \bar{\partial}-\partial \mu \partial)+\partial\left[\mu \bar{a}_{0}+A_{0}^{\prime 0,1}-\mu \bar{A}_{0}^{\prime 1,0}\right]+\right.$ (conjugate $)^{-1}$. 
(f)

$$
\begin{aligned}
& \sigma \cdot \operatorname{det}(\operatorname{det})^{*} \\
& =\exp (-S(\mu) F \cdot F) \\
& \quad \cdot \exp \left(\frac{i}{2 \pi} \int(\partial \bar{\partial}-\partial \mu \partial)^{-1}\left(\partial\left[\mu \bar{a}_{0}+A_{0}^{\prime 0,1}-\mu \bar{A}_{0}^{\prime 1,0}\right]\right) \wedge F\right) \\
& \quad \cdot \exp \left(\frac{i}{2 \pi} \int\left(a_{0}^{\prime} \wedge \bar{a}_{0}^{\prime}+a_{0}+\bar{a}_{0}\right)\right) \Theta^{\prime}\left(a_{0}^{\prime}\right) \bar{\Theta}\left(a_{0}\right),
\end{aligned}
$$

where $S(\mu)=\frac{i}{2 \pi} *(\partial \bar{\partial}-\partial \mu \partial)^{-1}$.

(h) The real and imaginary parts of $S(\mu)$ are symmetric, with respect to the real Hilbert space structure (2.4).

(i) $\mathbb{R} e(S(\mu))=i *\left(\partial^{\prime} \bar{\partial}^{\prime}\right)^{-1}=2 * *^{\prime} \Delta^{\prime-1}$ (on 2-forms); hence if $\sigma \in S$ Diff, $\mathbb{R} e S(\mu)=2 \Delta^{\prime-1}$.

Proof of $(4.21)$. If we view $a$ as the projection $\left(\Omega^{1}\right)^{\mathbb{C}} \rightarrow \Omega^{0,1}$, then using (1.10),

$$
\begin{aligned}
a^{\prime} & =\sigma_{*} \circ a \circ \sigma^{*}=\left(\begin{array}{ll}
A(\sigma) & B(\sigma) \\
\bar{B}(\sigma) & \bar{A}(\sigma)
\end{array}\right)\left(\begin{array}{ll}
1 & \\
& 0
\end{array}\right)\left(\begin{array}{ll}
A\left(\sigma^{-1}\right) & B\left(\sigma^{-1}\right) \\
\bar{B}\left(\sigma^{-1}\right) & \bar{A}\left(\sigma^{-1}\right)
\end{array}\right) \\
& =\left(\begin{array}{cc}
A(\sigma) A^{*}(\sigma) & -A(\sigma) \bar{B}^{*}(\sigma) \\
\bar{B}(\sigma) A^{*}(\sigma) & -\bar{B}(\sigma) \bar{B}^{*}(\sigma)
\end{array}\right) \\
& =\left(\begin{array}{cc}
(1-\mu \bar{\mu})^{-1} & -(1-\mu \bar{\mu})^{-1} \mu \\
\bar{\mu}(1-\mu \bar{\mu})^{-1} & -\bar{\mu}(1-\mu \bar{\mu})^{-1} \mu
\end{array}\right) \\
& =\left(\begin{array}{cc}
1 & \\
\bar{\mu} & 1
\end{array}\right)\left(\begin{array}{rr}
(1-\mu \bar{\mu})^{-1} & \\
& 0
\end{array}\right)\left(\begin{array}{cc}
1 & -\mu \\
& 1
\end{array}\right) .
\end{aligned}
$$

If we apply this to a one-form of the form $d f=\bar{\partial} f+\partial f$, then we obtain (a) and (b). If we apply this to a one-form of the form $A=a-\bar{a}$, then we obtain (c).

Similarly, if we view $a_{0}$ as the projection onto $\operatorname{ker}\left(\partial: \Omega^{0,1} \rightarrow \Omega^{2}\right)$, then

$$
a_{0}^{\prime}=\sigma_{*} \circ a_{0} \circ \sigma^{*}=\left(\begin{array}{ll}
A(\sigma) a_{0} A^{*}(\sigma) & -A(\sigma) a_{0} \bar{B}^{*} \\
\bar{B}(\sigma) a_{0} A^{*}(\sigma) & -\bar{B}(\sigma) a_{0} \bar{B}^{*}
\end{array}\right) .
$$

If $\sigma \in \operatorname{Aut}(\Sigma)$, then $\sigma \circ a_{0} \circ \sigma^{*}=a_{0}$; this implies $(c)^{\prime}$ (although we would dearly love to have an explicit formula).

Assuming that $x$ is real, as in the proof of (2.13),

$$
\begin{aligned}
a & =\bar{\partial} x+a_{0}=(1+\mu \text { conj })^{-1}(\bar{\partial}-\mu \partial) x^{\prime}+\left(a_{0}^{\prime}-\bar{a}_{0}^{\prime}\right)^{0,1} \\
& \Longrightarrow(\bar{\partial}+\mu \partial) x+a_{0}+\mu \bar{a}_{0}=(\bar{\partial}-\mu \partial) x^{\prime}+A_{0}^{\prime 0,1}-\mu A_{0}^{\prime 1,0} .
\end{aligned}
$$

By applying $\partial$ to both sides and solving for $x^{\prime}$, we obtain (d).

The other parts follow as in the proof of (2.14). 
Given $\sigma \in S$ Diff, we define

$$
\begin{aligned}
c_{T}(\sigma)= & \exp \left(-T(S(\mu)-S(0))_{\mathrm{reg}}+T L\left(\mu, a_{0}\right) \cdot_{T} F\right) \\
& \cdot \exp \left(\frac{i}{2 \pi} \int\left(a_{0}^{\prime} \wedge \bar{a}_{0}^{\prime}-a_{0} \wedge \bar{a}_{0}\right)\right) \frac{\Theta^{\prime}}{\Theta}
\end{aligned}
$$

(a random variable with respect to $\nu_{T}$ ), where

$$
L\left(\mu, a_{0}\right)=\frac{i}{2 \pi} *(\partial \bar{\partial}-\partial \mu \partial)^{-1}\left(\partial\left[\mu a_{0}+A_{0}^{\prime 0,1}-\mu A_{0}^{\prime 1,0}\right]\right)
$$

(a smooth two-form with vanishing integral). As in (2.29), $c_{T}$ is an SDiffcocycle with respect $\nu_{T}$.

We define the measure $d \nu_{T}^{|\mathcal{L}|^{-2}}$ having values in the line bundle $|\mathcal{L}|^{-2}$ by

$$
s_{1} \bar{s}_{2} d \nu_{T}^{|\mathcal{L}|^{-2}}=\frac{s_{1}}{\operatorname{det}}\left(\frac{s_{2}}{\operatorname{det}}\right)^{*} e^{-T S(0)_{\operatorname{reg}}} e^{\frac{i}{2 \pi} \int a_{0} \wedge \bar{a}_{0}}|\Theta|^{2} d \nu_{T},
$$

where $s_{1}, s_{2}$ are sections of $\mathcal{L}$ over the support of $\nu_{T}$. We then have (2.31), and we can define the representation $\pi_{T}$. The spherical function is given by

$$
\begin{aligned}
& \phi_{T}(\sigma)=\left\langle\pi_{T}(\sigma) \cdot \operatorname{det}, \operatorname{det}\right\rangle=\int \sigma \cdot \operatorname{det}(\operatorname{det})^{*} d \nu_{T}^{|\mathcal{L}|^{-2}} \\
& =\int e^{-T S_{\mathrm{reg}}+T L \cdot_{T} F-\frac{1}{4 \pi}\left(\left|A_{0}^{\prime}\right|^{2}+\left|A_{0}\right|^{2}\right)} \Theta^{\prime} \bar{\Theta} d \nu_{T} \\
& =\operatorname{det}\left((1+2 T S) e^{-2 T S}\right)^{-1 / 2} \int e^{\frac{T}{2}(1+2 T S)^{-1} L \cdot L} e^{-\frac{1}{4 \pi}\left(\left|A_{0}^{\prime}\right|^{2}+\left|A_{0}\right|^{2}\right)} \Theta^{\prime} \bar{\Theta}
\end{aligned}
$$

where $S=S(\mu), L=L\left(\mu, A_{0}\right),\left|A_{0}\right|^{2}=-\int A_{0} \wedge * A_{0}$, and the integral is with respect to the translation invariant measure on the torus $H^{1}(\Sigma, \mathbb{T})$.

Proposition 4.29. In terms of the notations of the preceding paragraph, the limit

$$
\begin{aligned}
& \lim _{T \rightarrow \infty} \frac{\phi_{T}(\sigma)}{\phi_{T}(0)} \\
& =\frac{\operatorname{det}_{\zeta}(4 \Delta)^{1 / 2}}{\operatorname{det}_{\zeta}\left(2 \Delta^{2}(\partial \bar{\partial}-\partial \mu \partial)^{-1}\right)^{1 / 2}} \int e^{2 \pi i *(\partial \bar{\partial}-\partial \mu \partial) L \cdot L} e^{-\frac{1}{4 \pi}\left(\left|A_{0}^{\prime}\right|^{2}+\left|A_{0}\right|^{2}\right)} \Theta^{\prime} \bar{\Theta}
\end{aligned}
$$

exists and defines a positive definite function on SDiff.

This follows from (3.2). 


\section{References}

[C] A. Connes, The Dixmier trace and the Wodzicki residue, preprint; incorporated into Noncommutative Geometry, Academic Press, 1994.

[G] K. Gawedzki, Lectures on conformal field theory, School of Mathematics, IAS, Princeton, 1996, to appear.

[I] R.S. Ismagilov, Representations of Infinite-Dimensional Groups, AMS Translations of Mathematical Monographs, 152 (1996).

[K] A. Knapp, Representation Theory of Semisimple Groups, Princeton University Press, 1986.

[KV] M. Kontsevich and S. Vishik, Geometry of determinants of elliptic operators, in Functional Analysis on the Eve of the 21st Century, 1 (New Brunswick, NJ, 1993), Prog. Math., 131, Birkhauser, (1995), 173-197.

[L] O. Lehto, Univalent Functions and Teichmuller Spaces, Springer-Verlag, 1986.

[Pi] D. Pickrell, On YM $M_{2}$ measures and area-preserving diffeomorphisms, J. Geom. and Physics, 19 (1996), 315-367.

[R] R. Ramer, On nonlinear transformations of Gaussian measures, J.F.A., 15 (1974), 166-187.

[Sen] A. Sengupta, Gauge theory on compact surfaces, Memoirs of the AMS, 126(600) (1997).

[Sh] M.A. Shubin, Pseudodifferential Operators and Spectral Theory, Springer-Verlag, 1987.

Received December 1, 1997 and revised March 10, 1999.

UNIVERSITY OF ARIZONA

TuCsON, AZ 85721

E-mail address: Pickrell@math.arizona.edu 